\title{
BMJ Open Investigating and addressing the immediate and long-term consequences of the COVID-19 pandemic on patients with substance use disorders: a scoping review and evidence map protocol
}

\author{
Leen Naji (D) , ${ }^{1,2}$ Brittany Dennis, ${ }^{3}$ Rebecca L Morgan (D) , ${ }^{2}$ Nitika Sanger (D) , ${ }^{4}$ \\ Andrew Worster, ${ }^{2,3}$ James Paul, ${ }^{5}$ Lehana Thabane (1) , 2,6 Zainab Samaan (1) ${ }^{2,7}$
}

To cite: Naji L, Dennis B, Morgan RL, et al. Investigating and addressing the immediate and long-term consequences of the COVID-19 pandemic on patients with substance use disorders: a scoping review and evidence map protocol. BMJ Open 2021;11:e045946. doi:10.1136/ bmjopen-2020-045946

- Prepublication history for this paper is available online. To view these files, please visit the journal online (http://dx.doi. org/10.1136/bmjopen-2020 045946).

Received 16 0ctober 2020 Accepted 09 August 2021
Check for updates

(C) Author(s) (or their employer(s)) 2021. Re-use permitted under CC BY-NC. No commercial re-use. See rights and permissions. Published by BMJ.

For numbered affiliations see end of article.

Correspondence to

Dr Leen Naji;

leen.naji@medportal.ca

\section{ABSTRACT}

Introduction The COVID-19 pandemic has driven unprecedented social and economic reform in efforts to curb the impact of disease. Governments worldwide have legislated non-essential service shutdowns and adapted essential service provision in order to minimise face-to-face contact. We anticipate major consequences resulting from such policies, with marginalised populations expected to bear the greatest burden of such measures, especially those with substance use disorders (SUDs). Methods and analysis We aim to conduct (1) a scoping review to summarise the available evidence evaluating the impact of the COVID-19 pandemic on patients with SUDs, and (2) an evidence map to visually plot and categorise the current available evidence evaluating the impact of COVID-19 on patients with SUDs to identify gaps in addressing high-risk populations.

Ethics and dissemination Ethics approval is not required for this scoping review as we plan to review publicly available data. This is part of a multistep project, whereby we intend to use the findings generated from this review in combination with data from an ongoing prospective cohort study our team is leading, encompassing over 2000 patients with SUDs receiving medication-assisted therapy in Ontario prior to and during the COVID-19 pandemic.

\section{INTRODUCTION}

The COVID-19 pandemic has driven unprecedented social and economic reform in efforts to curb the impact of disease. Governments worldwide have legislated nonessential service shutdowns and adapted essential service provision in order to minimise face-to-face contact. Despite reducing disease burden, we anticipate major consequences resulting from such policies, ${ }^{1-3}$ with marginalised populations expected to bear the greatest burden of such measures, especially those with substance dependence. We are already seeing increased rates of opioid overdoses across Canada.
Strengths and limitations of this study

- This will be the first scoping review and evidence map evaluating the impacts of COVID-19 on patients with substance use disorders.

- This review will address both classically measured treatment outcomes (eg, retention in treatment) and patient-important outcomes (eg, job stability).

- The utilisation of novel methods, namely evidence mapping, allows for user-friendly visual presentation of our findings.

- The combined expertise of our scientific team (including methodologists, statisticians, clinical addiction experts and guideline developers) will ensure wider uptake of evidence gained from this study.

- Our evidence map will allow us to develop efficient pragmatic strategies that tailor treatment intensity based on risk stratification, an essential component of pragmatic guidelines given the limited resources and financial strain of the healthcare system.

Patients with substance use disorders (SUDs) exhibit higher rates of mental illness, ${ }^{4}$ overdose, ${ }^{5}$ poly-substance use ${ }^{6}$ homelessness ${ }^{7}$ and financial instability ${ }^{7}$-all of which independently puts them at a disadvantage during a pandemic. ${ }^{8}$ From the exacerbation of mental illness stemming from social isolation, to increased COVID-19 transmission among populations with transient housing, we foresee the potential harmful effects to disproportionally impact patients with SUDs. Additionally, the shift to virtual care delivery for outpatient management of SUDs may prove less accessible among patients of low socioeconomic status who may not have access to an electronic device. To make matters worse, some integral services for maintaining sobriety including structured peer support programmes such as Alcoholics Anonymous ${ }^{9}$ 
have been altogether stopped, leaving a large number of patients vulnerable to relapse and overdose. Additionally, as governing bodies have advised physicians to loosen prescription practices for methadone and buprenorphine, patients are being less supervised in treatment and having less contact with their care team. Patients are no longer providing routine urine samples for toxicology screening given the limitations of telemedicine, and are instead receiving more take-home doses to minimise the risk of contracting the virus at the pharmacy while going in for a routine observed dose. The stress of the pandemic and social isolation itself, compounded by the lack of close follow-up and peer-support therapy, an integral part of treatment, is certainly going to have detrimental effects on these patients' health and well-being. As such, we anticipate the magnitude of serious epidemics, such as the opioid crisis, to worsen in the wake of COVID-19. ${ }^{10}$

In addition to being especially affected due to their social context and SUD, they are also at higher risk to suffer deleterious consequences from contracted COVID19. This is because respiratory illnesses (ie, COVID-19) compromise one's respiratory reserve thus putting them at higher risk of respiratory depression and death from sedating substances, including opioids and benzodiazepines. ${ }^{11}$ This is exceedingly concerning given social separation practices will mean that individuals using opioids, be it prescribed or illicit, will often be unsupervised and thus less likely to have someone administer the lifesaving antidote, naloxone. ${ }^{1}$ Unintentional overdose is already the leading cause of accidental death in the USA. ${ }^{12}$ This is a particular concern during the pandemic with expected increase in all-cause mortality. Taken together, the compounded deleterious consequences of this population's mental, physical and social predispositions easily render them the most disadvantaged during this crisis.

\section{STUDY OBJECTIVES}

We aim to conduct (1) a scoping review to summarise the available evidence evaluating the impact of the COVID-19 pandemic on patients with SUDs and (2) an evidence map to visually plot and categorise the current available evidence evaluating the impact of COVID-19 on patients with SUDs to identify gaps in addressing high-risk populations. We focus this review on the COVID-19 pandemic in light of the unique policies affecting social separation and health service delivery which have never been imposed previously.

Where possible, we aim to stratify associations by sex differences. Our team has previously shown that men and women are affected by substance use differently, ${ }^{13-16}$ therefore we would expect the impacts of social isolation and economic shut down to affect men and women uniquely. Are the previously established sex-driven differential health outcomes maintained or potentially magnified during COVID-19? Given women more likely suffer from depression, ${ }^{17-19}$ which may be exacerbated by social separation, are they at higher risk of overdose than men during this time? These questions highlight important areas requiring investigation to help appropriately tailor our delivery of sex and gender aware safe treatment schedules.

\section{METHODS}

The scoping review will be conducted as per published guidelines, ${ }^{20}$ and provides a summary of the available evidence evaluating the impact of COVID-19 on patients with SUDs. We are adapting traditional evidence mapping methodology usually applied to systematic reviews of direct comparison interventions to address risk factors and prognosticators during the COVID-19 pandemic. ${ }^{21} 22$ The evidence map is a relatively recent study design that provides a bird's-eye view of the landscape of available evidence, allowing us to identify trends and gaps in research using visual presentation. ${ }^{21} 22$ We will follow reporting guidelines as per the Preferred Reporting Items for Systematic Reviews and Meta-Analyses extension for Scoping Reviews. ${ }^{23}$

The following databases will be searched from December 2019 (ie, first case of COVID-19) to present: OVID Medline, EMBASE, PsycINFO, Web of Science, Cochrane Central Register of Controlled Trials, WHO Trial Registry, ClinicalTrials.gov and CINAHL. The search strategy was developed in consultation with an experienced health sciences librarian, and included multiple search terms related to COVID- $19^{24}$ and SUD (table 1). We will use previously validated search filters for SUD used by the Cochrane Collaboration. Additionally, we will manually search reference lists of included studies for any relevant missed articles. The search will be limited to human studies. No demographic, geographic or language constraints will be placed.

Titles, abstracts and full texts will be screened for relevance by two reviewers independently and in duplicate. Disagreements between reviewers will be resolved by consensus, or by consulting a third reviewer if necessary. Studies will be included if they meet the following eligibility criteria: (1) peer-reviewed primary studies, (2) among persons with SUDs as defined by classification systems (the Diagnostic and Statistical Manual of Mental Disorders, fifth edition ${ }^{25}$ and International Classifications of Diseases ${ }^{26}$ and (3) reporting on the effect of the COVID-19 pandemic on patient well-being, regardless of how it is defined by authors.

\section{Stage 2: data collection}

A standardised data collection chart will be developed by the research team. It will be pilot tested a priori in order to ensure that it is accurately capturing the necessary information. Two reviewers will independently manually extract the following data: author, year of publication, journal name, country, study design, sample size, study setting, participant demographics including age, sex, marital and employment status, type of SUD, intravenous drug use history, HIV and hepatitis C status, COVID-19 


\section{Table 1 Complete search terms}

Coronavirus (((((Coronavirus [MeSH Major Topic]) OR Coronavirus Infections [MeSH Major Topic]) OR covid-19) OR coronavirus) OR covid-19) OR $\left(\mathrm{nCoV}^{*}\right.$ or $2019 \mathrm{nCoV}$ or $19 \mathrm{nCoV}$ or covid-19* or covid-19 or SARS-COV-2 or SARSCOV-2 or SARSCOV2 or Severe Acute Respiratory Syndrome Coronavirus 2 or Severe Acute Respiratory Syndrome Corona Virus 2) OR ((new or novel or "19" or "2019" or Wuhan or Hubei or China or Chinese) adj3 (coronavirus* or corona virus* or betacoronavirus* or $\mathrm{CoV}$ or $\mathrm{HCoV}))$ )

\begin{tabular}{|c|c|}
\hline $\begin{array}{l}\text { Substance } \\
\text { use disorder }\end{array}$ & 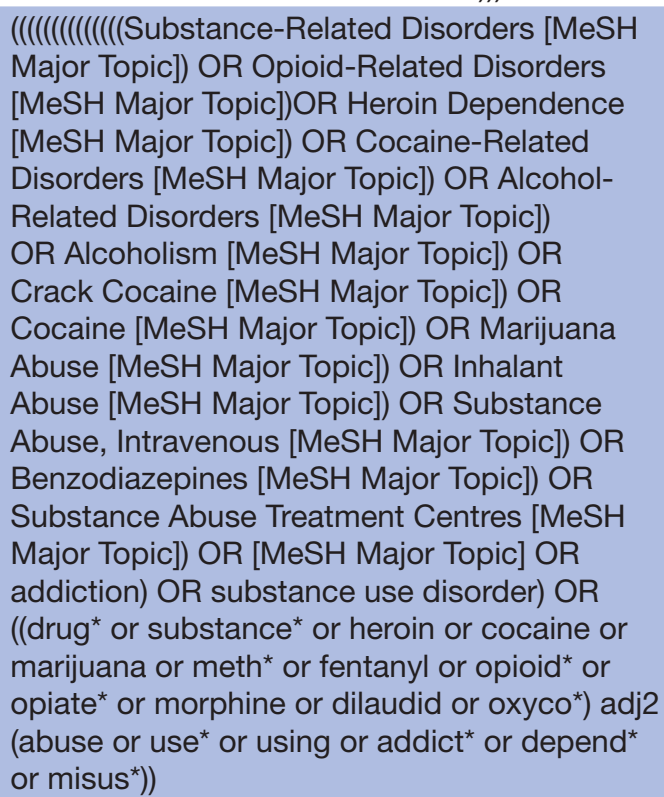 \\
\hline
\end{tabular}

MeSH, Medical Subject Headings.

status, retention in treatment, continued substance use and any other outcomes assessed in the study. Outcomes will be coded as being patient important or not, as what was previously defined among a substance use population. ${ }^{27}$ For the purpose of evidence mapping, data will be grouped according to outcome, prognostic factors and whether outcomes selected were patient-important.

\section{Stage 3: reporting the results}

Results will be summarised narratively in text and tables that were designed a priori. We will generate an evidence gap map using the 3ie EGM software ${ }^{28}{ }^{29}$ Where sufficient data exist, we will graphically display findings using a three-dimensional evidence gap map by outcome ( $y$-axis; $\mathrm{eg}$, retention in treatment), prognostic factors (x-axis; eg, unemployment) and patient preferences (z-axis; patient important outcome (yes/no)). Where possible, findings will be plotted for men and women separately, as well as a third plot for all other non-binary identifications of sex, allowing the reader to visually appreciate the differential outcomes by sex, stratified by prognostic factors including social determinants of health (ie, the x-axis). Additionally, where possible, secondary analyses will be conducted whereby we plan to study each type of SUD separately. We summarise quantitative results but do not plan to perform statistical analyses as we acknowledge the heterogeneity in the included studies.

\section{Patient and public involvement}

As this is an exploratory scoping review, we are seeking to identify trends and gaps in current evidence. As such, patients or the public were not involved in the design or conduct of this protocol. However, on completion of this study, we hope to involve patients with SUDs and the public to use our findings in helping design strategies to enhance outcomes among patients with SUDs during times of a pandemic.

\section{DISCUSSION}

Our scoping review and evidence map will allow us to describe the impact of COVID-19 on patients with SUDs, identify prognostic factors of poor outcome and characterise research gaps to highlight high-risk subpopulations among substance users that are overlooked in research and clinical care. Evidence maps are a novel and efficient tool that allows the reader to visually appreciate trends in available literature and certain outcomes or populations that may be overlooked in the research or more negatively affected by the pandemic. We may find that the majority of research lies in a single quadrant of the evidence map, for instance, suggesting a particular population may have been neglected in research studies. We plan to use our findings alongside quantitative data we are collecting from an ongoing prospective cohort study (9 years, pre-COVID-19 and post-COVID-19) investigating predictors of treatment outcomes among patients with SUDs. Collectively, our findings will guide us in identifying areas for future research and developing cost-effective practice recommendations for physicians in the treatment of patients with SUD during COVID-19, which can be adapted globally for future unexpected natural disasters, pandemics or shutdowns. In addition to gaining a comprehensive understanding and addressing the current state of SUDs, we aim to capitalise on our expertise to project the downstream needs of this patient population to proactively formulate recommendations to strategically adapt our clinical practice to meet the anticipated surge in care demands that will persist even after the resolution of the pandemic. Our evidence map will allow us to develop efficient pragmatic strategies that tailor treatment intensity based on risk stratification, an essential component of pragmatic guidelines given the limited resources and financial strain of the healthcare system.

\section{Strengths and limitations}

Our study is strengthened by the use of novel research methods to synthesise data in a user-friendly manner using a 3D evidence map. We place a focus on patient-important outcomes and highlighting the proportion of research studies taking these into account. Our broad search and eligibility criteria will allow us to capture a large proportion of research studies on the topic of SUDs during these unprecedented 
times of the COVID-19 pandemic. Our study findings may be limited by the fact that we are likely to rely primarily on retrospectively collected data and smaller cohort studies, limiting our ability to claim causality. Additionally, given the widespread COVID-19 pandemic, studies will likely lack an comparator group. However, as this is an exploratory study, we plan to use the data to identify trends and gaps in current evidence, and interpret data in the context of our ongoing prospective cohort study, as aforementioned.

\section{Ethics and dissemination}

Ethics approval was not required for this scoping review as we plan to review publicly available data. This is part of a multistep project, whereby we intend to use the findings generated from this review in combination with data from an ongoing prospective cohort study our team is leading, encompassing over 2000 patients with opioid use disorder receiving medication-assisted therapy in Ontario prior to and during the COVID-19 pandemic. We plan to disseminate data through traditional peer-reviewed publications, conference presentations, national discussion boards of practising addiction medicine physicians, department rounds and clinical practice guidelines. The combined expertise of our scientific team (including methodologists, statisticians, clinical addiction experts and guideline developers) will ensure wider uptake of evidence gained from this study.

\section{Author affiliations}

${ }^{1}$ Department of Family Medicine, McMaster University, Hamilton, Ontario, Canada

${ }^{2}$ Department of Health Research Methods, Evidence, and Impact, McMaster University, Hamilton, Ontario, Canada

${ }^{3}$ Department of Medicine, McMaster University, Hamilton, Ontario, Canada

${ }^{4}$ Medical Science Gradaute Program, McMaster University, Hamilton, Ontario, Canada

${ }^{5}$ Department of Anesthesia, McMaster University, Hamilton, Ontario, Canada

${ }^{6}$ Biostatistics Unit, Research Institute at St Joseph's Healthcare, Hamilton, Ontario, Canada

${ }^{7}$ Psychiatry and Behavioral Neurosciences, McMaster University, Hamilton, Ontario, Canada

Contributors LN, BD, NS, ZS conceived the research question. LN, BD, RLM, NS, AW, JP, LT and ZS designed the review protocol. LN, BD and NS designed the search strategy, which was approved by a health sciences librarian. All authors contributed to the writing and revision of the manuscript. All authors approved the final version of the manuscript.

Funding The authors have not declared a specific grant for this research from any funding agency in the public, commercial or not-for-profit sectors.

Competing interests None declared.

Patient and public involvement Patients and/or the public were not involved in the design, or conduct, or reporting, or dissemination plans of this research.

Patient consent for publication Not required.

Provenance and peer review Not commissioned; externally peer reviewed.

Open access This is an open access article distributed in accordance with the Creative Commons Attribution Non Commercial (CC BY-NC 4.0) license, which permits others to distribute, remix, adapt, build upon this work non-commercially, and license their derivative works on different terms, provided the original work is properly cited, appropriate credit is given, any changes made indicated, and the use is non-commercial. See: http://creativecommons.org/licenses/by-nc/4.0/.

\section{ORCID iDs}

Leen Naji http://orcid.org/0000-0003-0994-1109
Rebecca L Morgan http://orcid.org/0000-0002-1012-4897

Nitika Sanger http://orcid.org/0000-0002-5883-1873

Lehana Thabane http://orcid.org/0000-0003-0355-9734

Zainab Samaan http://orcid.org/0000-0002-5974-9361

\section{REFERENCES}

1 Volkow ND. Collision of the COVID-19 and addiction epidemics. Ann Intern Med 2020;173:61-2.

2 Becker WC, Fiellin DA. When epidemics collide: coronavirus disease 2019 (COVID-19) and the opioid crisis. Ann Intern Med 2020;173:59-60.

3 Alexander GC, Stoller KB, Haffajee RL, et al. An epidemic in the midst of a pandemic: opioid use disorder and COVID-19. Ann Intern Med 2020;173:57-8.

4 Buckley PF, Miller BJ, Lehrer DS, et al. Psychiatric comorbidities and schizophrenia. Schizophr Bull 2009;35:383-402.

5 Elzey MJ, Barden SM, Edwards ES. Patient characteristics and outcomes in unintentional, non-fatal prescription opioid overdoses: a systematic review. Pain Physician 2016;19:215-28.

6 Fernández-Calderón D, Fernández F, Ruiz-Curado S, et al. Profiles of substance use disorders in patients of therapeutic communities: link to social, medical and psychiatric characteristics. Drug Alcohol Depend 2015;149:31-9.

7 Kertesz SG, Crouch K, Milby JB, et al. Housing first for homeless persons with active addiction: are we overreaching? Milbank $Q$ 2009;87:495-534.

8 Elswick A, Fallin-Bennett $\mathrm{A}$, Ashford $\mathrm{K}$, et al. Emerging adults and recovery capital: barriers and facilitators to recovery. J Addict Nurs 2018;29:78-83.

9 Gossop M, Stewart D, Marsden J. Attendance at narcotics anonymous and alcoholics anonymous meetings, frequency of attendance and substance use outcomes after residential treatment for drug dependence: a 5-year follow-up study. Addiction 2008;103:119-25.

10 Clay JM, Parker MO. Alcohol use and misuse during the COVID-19 pandemic: a potential public health crisis? Lancet Public Health 2020;5:e259.

11 Lambert DG. Opioids and the COVID-19 pandemic: does chronic opioid use or misuse increase clinical vulnerability? Br J Anaesth 2020;125:e382-3.

12 CDC Injury Center. Opioid overdose / drug overdose. Available: https://www.cdc.gov/drugoverdose/index.html [Accessed 26 Apr 2020].

13 Bawor M, Dennis BB, Samaan MC, et al. Methadone induces testosterone suppression in patients with opioid addiction. Sci Rep 2014;4:6189.

14 Bawor M, Bami H, Dennis BB, et al. Testosterone suppression in opioid users: a systematic review and meta-analysis. Drug Alcohol Depend 2015;149:1-9.

15 Bawor M, Dennis BB, Bhalerao A. Sex differences in outcomes of methadone maintenance treatment for opioid use disorder: a systematic reviewand meta-analysis. C Open 2015.

16 Bawor M, Dennis BB, Varenbut M, et al. Sex differences in substance use, health, and social functioning among opioid users receiving methadone treatment: a multicenter cohort study. Biol Sex Differ 2015;6:21.

17 Hser YI, Anglin MD, McGlothlin W. Sex differences in addict careers. 1. initiation of use. Am J Drug Alcohol Abuse 1987;13:33-57.

18 Anglin MD, Hser YI, McGlothlin WH. Sex differences in addict careers. 2. becoming addicted. Am J Drug Alcohol Abuse 1987;13:59-71.

19 Hser YI, Anglin MD, Booth MW. Sex differences in addict careers. 3. addiction. Am J Drug Alcohol Abuse 1987;13:231-51.

20 Peters MDJ, Godfrey C, McInerney P. Chapter 11: scoping reviews (2020 version). In: Aromataris E, Munn Z, eds. Joanna briggs institute reviewer's manual. JBI, 2020. https://reviewersmanual.joannabriggs. org

21 Miake-Lye IM, Hempel S, Shanman R, et al. What is an evidence MAP? A systematic review of published evidence maps and their definitions, methods, and products. Syst Rev 2016;5:28.

22 Snilstveit B, Vojtkova M, Bhavsar A. Evidence gap maps a tool for promoting evidence-informed policy and prioritizing future research, 2013. Available: http://econ.worldbank.org [Accessed 05 May 2020].

23 Tricco AC, Lillie E, Zarin W, et al. PRISMA extension for scoping reviews (PRISMA-ScR): checklist and explanation. Ann Intern Med 2018;169:467-73.

24 CADTH. CADTH COVID-19 search strings - CADTH Covid-19 evidence portal. Available: https://covid.cadth.ca/literaturesearching-tools/cadth-covid-19-search-strings/ [Accessed 15 Oct 2020]. 
25 American Psychiatric Association. Diagnostic and statistical manual of mental disorders: diagnostic and statistical manual of mental disorders. 5th Edn. Arlington, VA: American Psychiatric Association, 2013.

26 WHO. International classification of diseases (ICD-10). Fam Pract Manag 2010.

27 Dennis BB, Sanger N, Bawor M, et al. A call for consensus in defining efficacy in clinical trials for opioid addiction: combined results from a systematic review and qualitative study in patients receiving pharmacological assisted therapy for opioid use disorder. Trials 2020;21.

28 Snilstveit B, Bhatia R, Rankin K, et al. Working paper 28 3ie evidence gap maps a starting point for strategic evidence production and use, 2017.

29 Saran A, White H. Evidence and gap maps: a comparison of different approaches. Campbell Syst Rev 2018;14:1-38. 